\title{
PI OBSERVER DESIGN FOR A CLASS OF NONDIFFERENTIALLY FLAT SYSTEMS
}

\author{
JUAN PABLO FLORES-FLORES ${ }^{a}$, RAFAEL MARTINEZ-GUERRA $^{a, *}$
}

\author{
${ }^{a}$ Department of Automatic Control \\ CINVESTAV-IPN, Av. Instituto Politecnico Nacional 2508, La Laguna Ticoman, 07360 Mexico City, Mexico \\ e-mail: \{jflores, rguerra\}@etrl.cinvestav.mx
}

\begin{abstract}
This paper presents a methodology and design of a model-free-based proportional-integral reduced-order observer for a class of nondifferentially flat systems. The problem is tackled from a differential algebra point of view, that is, the state observer for nondifferentially flat systems is based on algebraic differential polynomials of the output. The observation problem is treated together with that of a synchronization between a chaotic system and the designed observer. Some basic notions of differential algebra and concepts related to chaotic synchronization are introduced. The PI observer design methodology is given and it is proven that the estimation error is uniformly ultimately bounded. To exemplify the effectiveness of the PI observer, some cases and their respective numerical simulation results are presented.
\end{abstract}

Keywords: model-free-base PI reduced-order observer, synchronization, nondifferentially flat system, algebraic observability condition, uniform ultimate boundedness.

\section{Introduction}

Most of control techniques assume a complete knowledge of parameters and state variables of the system at any time. Nevertheless, in practice this is not always possible due to physical limitations. Therefore, it is necessary to design observers for such variables. In the literature, the differential geometry approach has been widely used with excellent results (Gauthier et al., 1992). However, it is well known that differential algebra offers certain advantages with respect to the differential geometric approach (cf. Martínez-Guerra et al., 2015). For this reason, some authors have employed algebraic techniques to attack the observation problem (Martínez-Guerra et al., 2007).

Differential algebra theory was developed by Ritt (1950) in order to generalize classical algebra and to make it possible to study solutions in the context of ordinary and partial algebraic differential equations. Solutions described by nonlinear differential polynomials are particularly interesting. In general, differential algebra is said to be the field of mathematics that studies algebraic structures equipped with a derivation operation. Some algebraic structures are differential rings and differential

*Corresponding author fields.

In control theory, a fundamental concept for the observation problem is the observability property of a dynamical system. There exists an equivalent definition for the observability property in differential algebra terms. It is known as the algebraic observability condition (AOC) and was formulated in order to solve the state estimation problem of nonlinear dynamical systems through differential polynomials of the known variables (Kolchin, 1973; Martínez-Guerra and Cruz-Ancona, 2017). An unknown variable is said to be algebraically observable if it satisfies a differential polynomial of the known variables. A system satisfies the AOC if all unknown variables are algebraically observable.

On the other hand, differential flatness of a dynamical systems has huge relevance for control theory. This property has been widely used as an indicator of controllability presented by a dynamical system (Sira-Ramirez, 2002). Most of differential flatness applications are related to the trajectory planning for unmanned vehicles (Gensior et al., 2006; Pledgie et al., 2002). Roughly speaking, a dynamical system is said to be differentially flat if all its variables satisfy an algebraic differential equation of the output. Any system which does not satisfy it is said to be 
nondifferentially flat. Nondifferentially flat systems have received little attention. A few studies for this kind of systems are related to synchronization of chaotic systems (Martínez-Guerra et al., 2015).

One can note a relationship between the AOC and nondifferential flatness of a system. That is, any system that is nondifferentially flat, does not satisfy the AOC. However, in this paper it will be shown that there is a possibility to estimate unknown variables of a nondifferentially flat system from an auxiliary variable which, when selected in the right form, is algebraically observable. As far as we know, in the literature there is no methodology to solve the estimation problem of a nondifferentially flat system. Even more, the model-free-based PI reduced-order observer proposed here is a more natural way of setting up an observer since we usually do not have any exact copy of the system.

The methodology stated here requires, among other things, that the observed system have bounded dynamics. Therefore, it is proposed for chaotic systems. A problem related to chaotic systems, which is of great interest in nonlinear theory, is the synchronization problem. Basically, synchronization occurs when, given two coupled chaotic systems, their state trajectories are equal. Thus, the synchronization problem is analogous to the observation problem treated here. In this case, the chaotic system and the PI observer are unidirectionally coupled, where the output plays the role of the coupling signal and the synchronization phenomenon occurs when the estimation error is zero.

The methodology stated here is as follows. Given a nondifferentially flat dynamical system, an auxiliary variable is proposed. This variable is selected such that is a function of unknown variables and is algebraically observable. Given this auxiliary variable, the original system is expressed as an extended system, known as immersion (Claude et al., 1983). Thus, the original problem becomes an estimation problem of the auxiliary variable. Depending on the selection made, variables of interest are estimated directly or indirectly through the estimation of the auxiliary variable. To solve the estimation problem of the auxiliary variable, some hypotheses are needed: the auxiliary variable is algebraically observable and its dynamics are bounded. Besides, there exist artificial variables which are continuously differentiable. If these hypotheses are fulfilled, it is possible to design a PI observer. In order to exemplify the methodology and show its effectiveness, some examples and their respective numerical results are given.

This paper is an extended version of our previous work (Martinez-Guerra and Flores-Flores, 2018), and additional material is included.

\section{Basic definitions}

The main ingredient for the PI observer design is the algebraic observability property. In order to properly introduce this concept and some others of interest, such as a nondifferential flat system, in the following some basic notions and definitions are introduced.

Definition 1. Let $K$ and $L$ be differential fields. An element $x \in L$ is called differentially algebraic over $K$ if satisfies a polynomial differential equation $P\left(x, \dot{x}, \ldots, x^{(\alpha)}\right)=0$ with coefficients in $K$. In what follows, the field $K=\mathbb{R}$ is considered.

Example 1. Consider the linear system

$$
\begin{aligned}
\dot{x}_{1} & =x_{2}+u, \\
\dot{x}_{2} & =x_{1}, \\
y & =x_{1}
\end{aligned}
$$

such that

$$
\begin{aligned}
& P\left(x_{1}\right)=y-x_{1}=0, \\
& P\left(x_{2}\right)=\dot{y}-x_{2}-u=0
\end{aligned}
$$

with coefficients $u, y, \dot{y} \in K\langle u, y\rangle=K \cup\{u, y\}$ that represents a differential field and a set $\{u, y\}$ that contains $u, y$ and their finite differential quantities $(K \subset K\langle u, y\rangle)$, that are coefficients of the differential polynomials with indeterminate $x$.

Then consider the bilinear system

$$
\begin{aligned}
\dot{x}_{1} & =u x_{2}, \\
\dot{x}_{2} & =u x_{1}, \\
y & =x_{1}
\end{aligned}
$$

such that

$$
\begin{aligned}
& P\left(x_{1}\right)=y-x_{1}=0, \\
& P\left(x_{2}\right)=\dot{y}-u x_{2}=0
\end{aligned}
$$

with coefficients $u, y, \dot{y} \in K\langle u, y\rangle$.

Finally, consider the nonlinear system

$$
\begin{aligned}
\dot{x}_{1} & =u x_{2} x_{1}, \\
\dot{x}_{2} & =u x_{1}, \\
y & =x_{1}
\end{aligned}
$$

such that

$$
\begin{aligned}
& P\left(x_{1}\right)=y-x_{1}=0, \\
& P\left(x_{2}\right)=\dot{y}-u x_{2} y=0
\end{aligned}
$$

with coefficients $u, y, \dot{y} \in K\langle u, y\rangle$.

One can note that in all the three cases $x \in$ $\mathbb{R}^{2}$ satisfies a polynomial differential equation with coefficients in $K\langle u, y\rangle, K=\mathbb{R}$. Therefore, it is said to be differentially algebraic. 
Definition 2. A state variable $x_{i}$ of a dynamical system is said to be algebraically observable if it satisfies a differential polynomial with respect to known variables, i.e., $P\left(x_{i}\right)=0$ with coefficients in $K\langle u, y\rangle(K=$ $\mathbb{R})$. If all state variables of the dynamical system are algebraically observable, we say that the system satisfies an algebraic observability condition (AOC).

Definition 3. A dynamical system is said to be nondifferentially flat if any variable is not differentially algebraic. That is, the polynomial differential equation $P\left(x_{i}, u_{i}\right)=$ 0 with coefficients in $K\langle y\rangle(K=\mathbb{R})$ is not satisfied. Otherwise is said to be differentially flat.

Example 2. Consider the following Lorenz system:

$$
\begin{aligned}
\dot{x}_{1} & =\sigma\left(x_{2}-x_{1}\right), \\
\dot{x}_{2} & =\rho x_{1}-x_{2}-x_{1} x_{3}, \\
\dot{x}_{3} & =x_{1} x_{2}-\beta x_{3}, \\
y & =x_{1} .
\end{aligned}
$$

It follows that

$$
\begin{aligned}
& P\left(x_{1}\right)=y-x_{1}=0, \\
& P\left(x_{2}\right)=\sigma\left(x_{2}-y\right)-\dot{y}=0, \\
& P\left(x_{3}\right)=y\left(x_{3}-\rho+1\right)+\dot{y}\left(1+\frac{1}{\sigma}\right)+\frac{\ddot{y}}{\sigma}=0 .
\end{aligned}
$$

We can note that $P\left(x_{3}\right)$ is not rational since $y \neq 0$ and its derivatives are coefficients in $K\langle y\rangle$. Therefore, the system is said to be differentially flat.

On the other hand, consider $y=x_{2}$, such that

$$
\begin{aligned}
& P\left(x_{1}, \dot{x}_{1}\right)=\dot{x}_{1}-\sigma\left(y-x_{1}\right)=0, \\
& P\left(x_{2}\right)=y-x_{2}=0, \\
& P\left(x_{1}, x_{3}, \dot{x}_{3}\right)=\dot{x}_{3}+\beta x_{3}-y x_{1}=0 .
\end{aligned}
$$

In this case the system is nondifferentially flat, since Definition 3 is not satisfied.

Remark 1. It is possible to note a relation between the nondifferential flatness of a system and the AOC. For a nondifferentially flat system, the AOC is not fulfilled. For this reason it is not possible to build an observer for all variables of interest. This motivates the methodology proposed in this paper.

Definition 4. A chaotic system

$$
\dot{x}=f(x)
$$

is a deterministic nonlinear system that exhibits an apparently random behavior that satisfies the following properties:

(i) $f(x)$ is bounded. That is, for the map $f: X \rightarrow$ $X$, where $X$ is a closed invariant set, there exist an attracting set $D \subset X$ such that $\forall t \geq 0$ and $\forall x \in X$, $f(x) \subset D$. (ii) Sensitive dependence on the initial condition. That is, the map $f: X \rightarrow X$ is said to have sensitive dependence on initial conditions if there exists a $\delta>$ 0 such that for any $x \in X$ and any neighborhood $N_{\varepsilon}(x)=(x-\varepsilon, x+\varepsilon)$ of $x$, there exist $y \in N_{\varepsilon}(x)$ and an integer $k>0$ such that $\left|f^{k}(x)-f^{k}(y)\right|>$ $\delta>0$.

It is worth mentioning that chaotic behavior depends on system parameters as well. For further details, see the work of Layek (2015).

Definition 5. Consider two chaotic systems coupled unidirectionally, given by

$$
\Sigma_{m}:=\left\{\begin{array}{l}
\dot{x}_{m}=F_{m}\left(x_{m}, u_{m}\right), \\
y_{m}=h_{m}\left(x_{m}\right)
\end{array}\right.
$$

and

$$
\Sigma_{s}:=\left\{\begin{array}{l}
\dot{x}_{s}=F_{s}\left(x_{s}, u_{s}\left(x_{s}, y_{m}\right)\right), \\
y_{s}=h_{s}\left(x_{s}\right) .
\end{array}\right.
$$

Systems $\Sigma_{m}$ and $\Sigma_{s}$ are synchronized if their state trajectories follow a common path.

Define the synchronization error as

$$
e_{\text {synch }}(t)=x_{m}-x_{s} .
$$

Hence systems $\Sigma_{m}$ and $\Sigma_{s}$ are synchronized if

$$
\lim _{t \rightarrow \infty}\left\|e_{\text {synch }}(t)\right\|=0 .
$$

In our case, the chaotic system and the observer are coupled unidirectionally by the output (Neijmeijer and Mareels, 1997).

\section{Problem statement}

In the following, consider the following dynamical system whose full state is not available:

$$
\begin{aligned}
& \dot{x}=F(x, u), \\
& y=h(x),
\end{aligned}
$$

where $x=\left[x_{1}, x_{2}, \ldots, x_{n}\right]^{T}$ is the state vector of the system, $F$ is an analytic function, $h$ is a polynomial. Variables $u=\left[u_{1}, u_{2}, \ldots, u_{m}\right]^{T}$ and $y \in \mathbb{R}$ are the input and output, respectively.

Define an auxiliary variable $\eta$ as a function of the unknown variables of the system. Thus, system (1) can be expressed as an immersion (see Claude et al., 1983). Therefore, we have

$$
\begin{aligned}
\dot{x}(t) & =f(x, \eta, u), \\
\dot{\eta}(x) & =\Omega(x), \\
y(t) & =h(x) .
\end{aligned}
$$


Remark 2. One can note that for (2), the problem is to estimate $\eta$. Depending on the selection of $\eta$, variables of interest are determined directly or indirectly. If $\eta$ is defined as $\eta=\left[\eta_{1}, \ldots, \eta_{\mu}\right]$, a bank of observers is proposed, that is, an observer is designed for each variable of interest.

3.1. Observer synthesis. Assume that the following hypotheses are fulfilled:

H1. $\eta$ is algebraically observable,

H2. $\Omega(x)$ is bounded, i.e., $\|\Omega(x)\| \leq N$, where $0<N<$ $\infty$,

H3. $\gamma_{1}, \gamma_{2} \in C^{1}$ real valued functions.

Then the following model-free-based proportional-integral reduced-order observer is proposed for the unknown dynamics of system (2), in this case $\dot{\eta}$ :

$$
\begin{aligned}
\dot{\hat{\eta}} & =k_{p}(\eta-\hat{\eta})+\hat{\eta}_{1}, \\
\dot{\hat{\eta}}_{1} & =k_{i}(\eta-\hat{\eta}),
\end{aligned}
$$

where $\hat{\eta}$ is the estimate of $\eta$ and $\hat{\eta}_{1}$ is the integral part of the observer. Define the estimation error as

$$
e=\eta-\hat{\eta}
$$

Theorem 1. Assume that $H 1-H 3$ are satisfied. Then system (3) is a model-free-based PI reduced-order observer for unknown dynamics of system (2) with a uniformly ultimately bounded (UUB) convergence error.

Proof. System (3) is equivalent to

$$
\dot{\hat{\eta}}=k_{p} e_{1}+k_{i} e_{2}
$$

where $e_{1}=\eta-\hat{\eta}$ and $\dot{e}_{2}=e_{1}$. Set

$$
E=\left(\begin{array}{l}
e_{1} \\
e_{2}
\end{array}\right)
$$

The derivative of $E$ is

$$
\begin{aligned}
\dot{E} & =\left(\begin{array}{c}
\dot{\eta}-\dot{\hat{\eta}} \\
e_{1}
\end{array}\right) \\
& =\left(\begin{array}{c}
\Omega-k_{p} e_{1}-k_{i} e_{2} \\
e_{1}
\end{array}\right) \\
& =-\left(\begin{array}{cc}
k_{p} & k_{i} \\
-1 & 0
\end{array}\right)\left(\begin{array}{c}
e_{1} \\
e_{2}
\end{array}\right)+\left(\begin{array}{c}
\Omega \\
0
\end{array}\right),
\end{aligned}
$$

or, in matrix form,

$$
\dot{E}=-K E+\bar{\Omega} .
$$
function:

Consider the following candidate Lyapunov

$$
V(E)=E^{T} P E
$$

with $P=I$. The derivative of $V(E)$ along the trajectories of (8) is given by

$$
\begin{aligned}
\dot{V}(E) & =\dot{E}^{T} E+E^{T} \dot{E} \\
& =2 E^{T} \dot{E} \\
& =2 E^{T}(-K E+\bar{\Omega}) \\
& =-2 E^{T} K E+2 E^{T} \bar{\Omega} .
\end{aligned}
$$

From the Rayleigh inequality we know that

$$
\lambda_{\min }(K)\|E\|^{2} \leq\|E\|_{K}^{2} \leq \lambda_{\max }(K)\|E\|^{2}
$$

such that the first term of $\dot{V}(E)$ is bounded by

$$
-2 E^{T} K E \leq-2 \lambda_{\min }(K)\|E\|^{2}
$$

On the other hand, since $\mathrm{H} 2$ is satisfied, $\bar{\Omega}$ is bounded. We have

$$
\left\|2 E^{T} \bar{\Omega}\right\| \leq 2 N\|E\|_{K}=2 N \sqrt{\lambda_{\max }(K)}\|E\| .
$$

Hence we conclude that $\dot{V}(E)$ satisfies

$$
\dot{V}(E) \leq-2 \lambda_{\min }(K)\|E\|^{2}+2 N \sqrt{\lambda_{\max }(K)}\|E\| .
$$

By applying the uniform ultimate boundedness (see Corless and Leitmann,1981), it directly follows that $E(t)$ is bounded uniformly for any initial condition $E(0)$, and $E(t)$ remains in the compact set $B_{\delta}=$ $\{E:\|E\| \leq \delta, \delta>0\}$, where

$$
\delta=\sqrt{\frac{\lambda_{\max }(K)}{\lambda_{\min }(K)}}\left(\frac{2 N \sqrt{\lambda_{\max }(K)}}{\lambda_{\min }(K)}\right)>0 .
$$

From 15 one can find a criterion to select the gains $k_{p}$ and $k_{i}$. We have

$$
\frac{\lambda_{\max }(K)}{\lambda_{\min }(K)} \frac{4 N^{2} \lambda_{\max }(K)}{\lambda_{\min }^{2}(K)}=\frac{4 N^{2} \lambda_{\max }^{2}(K)}{\lambda_{\min }^{3}(K)}>0 .
$$

To satisfy this last inequality, we must have $\lambda_{\min }(K)>0$ and thus $\lambda_{\max }(K)>0$.

Sometimes, depending on the selection of $\eta$, output derivatives might appear in the algebraic differential polynomial. As output derivatives are not available, it is necessary to use artificial variables in order to avoid them.

Lemma 1. If the auxiliary variable $\eta_{i}, i \in\{1, \ldots, \mu\}$, of system (2) satisfies $H 1$ and can be written as

$$
\eta_{i}=a_{i} \dot{y}+b_{i}(u, y)
$$

where $a_{i}$ is constant and $b_{i}(u, y)$ is a bounded function, then there exist functions $\gamma_{1_{i}}$ and $\gamma_{2_{i}} \in C^{1}$ such that the 
model-free-based PI reduced-order observer (3) can be expressed as

$$
\begin{aligned}
\dot{\gamma}_{1_{i}}= & -k_{p_{i}} \gamma_{1_{i}}+\gamma_{2_{i}}+k_{p_{i}}\left[b_{i}(u, y)-k_{p_{i}} a_{i} y\right] \\
& +k_{i_{i}} a_{i} y \\
\gamma_{1_{i}}(0)= & \gamma_{1 i_{0}} \\
\dot{\gamma}_{2_{i}}= & -k_{i_{i}} \gamma_{1_{i}}+k_{i_{i}}\left[b_{i}(u, y)-k_{p_{i}} a_{i} y\right] \\
\gamma_{2_{i}}(0)= & \gamma_{2 i_{0}} \\
\hat{\eta}_{i}= & \gamma_{1_{i}}+k_{p_{i}} a_{i} y \\
\hat{\eta}_{1_{i}}= & \gamma_{2_{i}}+k_{i_{i}} a_{i} y
\end{aligned}
$$

Proof. Consider the model-free-based PI reduced-order observer

$$
\begin{aligned}
\dot{\hat{\eta}}_{i} & =k_{p_{i}}\left(\eta_{i}-\hat{\eta}_{i}\right)+\hat{\eta}_{1_{i}}, \\
\dot{\hat{\eta}}_{1_{i}} & =k_{i_{i}}\left(\eta_{i}-\hat{\eta}_{i}\right),
\end{aligned}
$$

where $\hat{\eta}_{1_{i}}$ represents the integral part of the PI observer. Substituting (17) in 19, we have

$$
\begin{aligned}
\dot{\hat{\eta}}_{i} & =k_{p_{i}}\left(a_{i} \dot{y}+b_{i}(u, y)-\hat{\eta}_{i}\right)+\hat{\eta}_{1_{i}}, \\
\dot{\hat{\eta}}_{1_{i}} & =k_{i_{i}}\left(a_{i} \dot{y}+b_{i}(u, y)-\hat{\eta}_{i}\right) .
\end{aligned}
$$

We define the artificial variables $\gamma_{1_{i}}$ and $\gamma_{2_{i}} \in C^{1}$ as

$$
\begin{aligned}
& \gamma_{1_{i}}=\hat{\eta}_{i}-k_{p_{i}} a_{i} y, \\
& \gamma_{2_{i}}=\hat{\eta}_{1_{i}}-k_{i_{i}} a_{i} y .
\end{aligned}
$$

Their derivatives are

$$
\begin{aligned}
& \dot{\gamma}_{1_{i}}=\dot{\hat{\eta}}_{i}-k_{p_{i}} a_{i} \dot{y}, \\
& \dot{\gamma}_{2_{i}}=\dot{\hat{\eta}}_{1_{i}}-k_{i_{i}} a_{i} \dot{y} .
\end{aligned}
$$

From (20)-22) we have

$$
\begin{aligned}
\dot{\gamma}_{1_{i}}= & -k_{p_{i}} \gamma_{1_{i}}+\gamma_{2_{i}}+k_{p_{i}}\left[b_{i}(u, y)-k_{p_{i}} a_{i} y\right] \\
& +k_{i_{i}} a_{i} y \\
\gamma_{1_{i}}(0)= & \gamma_{1 i_{0}} \\
\dot{\gamma}_{2_{i}}= & -k_{i_{i}} \gamma_{1_{i}}+k_{i_{i}}\left[b_{i}(u, y)-k_{p_{i}} a_{i} y\right] \\
\gamma_{2_{i}}(0)= & \gamma_{2 i_{0}} .
\end{aligned}
$$

Remark 3. One can note that the proposed observer is of model-free, this is, an exact copy of the dynamical system is not required. The observer needs only the output of the system for its design.

3.2. PI observer implementation. The model-free PI observer methodology is illustrated with the following cases.
Example 3. Consider the linear system

$$
\begin{aligned}
\dot{x}_{1} & =u, \\
\dot{x}_{2} & =u+f, \\
y & =x_{2},
\end{aligned}
$$

where $u$ is known and $f$ is an unknown variable. The system (24) is nondifferentially flat due to

$$
\begin{aligned}
P\left(\dot{x}_{1}\right) & =\dot{x}_{1}-u=0, \\
P\left(x_{2}\right) & =x_{2}-y=0, \\
P(f) & =u+f-\dot{y}=0 .
\end{aligned}
$$

The linear system can be expressed in the following form (immersion):

$$
\begin{aligned}
\dot{x}_{1} & =u, \\
\dot{x}_{2} & =u+f, \\
\dot{f} & =\Omega\left(x_{1}, x_{2}\right), \\
y & =x_{2} .
\end{aligned}
$$

The PI observer is expressed as the following set of differential equations:

$$
\begin{aligned}
& \dot{\hat{f}}=k_{p}(f-\hat{f})+\hat{f}_{1}, \\
& \dot{\hat{f}}_{1}=k_{i}(f-\hat{f}),
\end{aligned}
$$

where $\hat{f}$ is the estimate of $f$ and $\hat{f}_{1}$ represents the integral part of the PI observer. One can check that $f$ satisfies H1, such that $f=\dot{y}-u$. Suppose that $\dot{f}$ is bounded (H2). Thus, substituting this in 27, we have

$$
\begin{aligned}
& \dot{\hat{f}}=k_{p}(\dot{y}-u-\hat{f})+\hat{f}_{1}, \\
& \dot{\hat{f}}_{1}=k_{i}(\dot{y}-u-\hat{f}) .
\end{aligned}
$$

As variable $\dot{y}$ is not auxiliary, we introduce some artificial variables that satisfy $\mathrm{H} 3$ in order to deal with this. Consider the following auxiliary variables:

$$
\begin{aligned}
\gamma_{1} & =\hat{f}-k_{p} y, \\
\gamma_{2} & =\hat{f}_{1}-k_{i} y .
\end{aligned}
$$

Deriving the auxiliary variables and replacing $\hat{f}$ and $\hat{f}_{1}$, we have

$$
\begin{aligned}
& \dot{\gamma}_{1}=-k_{p}\left(u+\gamma_{1}+k_{p} y\right)+\gamma_{2}+k_{i} y, \\
& \dot{\gamma}_{2}=-k_{i} u-k_{i}\left(\gamma_{1}+k_{p} y\right) .
\end{aligned}
$$

That is, the dynamics of interest are estimated from the auxiliary variables. Therefore, the model-free-based 
PI observer is given by

$$
\begin{aligned}
\dot{\gamma}_{1} & =-k_{p} \gamma_{1}+\gamma_{2}-k_{p}\left(u+k_{p} y\right)+k_{i} y, \\
\gamma_{1}(0) & =\gamma_{1_{0}} \\
\dot{\gamma}_{2} & =-k_{i} \gamma_{1}-k_{i}\left(u+k_{p} y\right), \\
\gamma_{2}(0) & =\gamma_{2_{0}} \\
\hat{f} & =\gamma_{1}+k_{p} y \\
\hat{f}_{1} & =\gamma_{2}+k_{i} y .
\end{aligned}
$$

Example 4. The Chua circuit was invented by Leon O. Chua. It is a simple electronic circuit that exhibits chaotic behaviour. The Chua circuit, also known as the Chua system, is basically it formed by two capacitors and an inductor. This system is described by three nonlinear differential equations and it was the first chaotic system proved to exist to exist by analytical and experimental means.

Consider the following Chua system:

$$
\begin{aligned}
\dot{x}_{1} & =a\left(x_{2}-x_{1}-m_{0} x_{1}-x_{1}^{3} m_{1}\right), \\
\dot{x}_{2} & =x_{1}-x_{2}+x_{3}, \\
\dot{x}_{3} & =-b x_{2}, \\
y & =x_{2},
\end{aligned}
$$

where $a, b, m_{0}$ and $m_{1}$ are constant parameters. Variables $x_{1}$ and $x_{2}$ correspond to the voltages across the capacitors and $x_{3}$ is the electric current in the inductor.

One can find that system (32) is nondifferentially flat due to

$$
\begin{aligned}
P\left(x_{1}, x_{3}\right) & =x_{1}-\dot{y}-y+x_{3}=0, \\
P\left(x_{2}\right) & =x_{2}-y=0, \\
P\left(\dot{x}_{3}\right) & =\dot{x}_{3}+b y=0 .
\end{aligned}
$$

We define the variable

$$
\eta=x_{1}+x_{3}=\dot{y}+y
$$

which satisfies H1. Therefore, system (32) is expressed in the following form (immersion):

$$
\begin{aligned}
\dot{x}_{1} & =a\left(x_{2}-x_{1}-m_{0} x_{1}-x_{1}^{3} m_{1}\right), \\
\dot{x}_{2} & =-x_{2}+\eta, \\
\dot{x}_{3} & =-b x_{2}, \\
\dot{\eta} & =\Omega\left(x_{1}, x_{3}\right), \\
y & =x_{2} .
\end{aligned}
$$

Since the Chua system dynamics are bounded, $\Omega\left(x_{1}, x_{3}\right)$ satisfies $\mathrm{H} 2$. The following PI observer is proposed in order to estimate $\eta$ :

$$
\begin{aligned}
\dot{\hat{\eta}} & =k_{p}(\dot{y}+y-\hat{\eta})+\hat{\eta}_{1}, \\
\dot{\hat{\eta}}_{1} & =k_{i}(\dot{y}+y-\hat{\eta}) .
\end{aligned}
$$

To remove the dependence on $\dot{y}$, consider the following auxiliary variables:

$$
\begin{aligned}
& \gamma_{1}=\hat{\eta}-k_{p} y, \\
& \gamma_{2}=\hat{\eta}_{1}-k_{i} y .
\end{aligned}
$$

From the derivatives of the auxiliary artificial variables we have

$$
\begin{aligned}
& \dot{\gamma}_{1}=k_{p}\left(y-\gamma_{1}-k_{p} y\right)+\gamma_{2}+k_{i} y \\
& \dot{\gamma}_{2}=k_{i}\left(y-\gamma_{1}-k_{p} y\right) .
\end{aligned}
$$

Therefore, the model-free-based PI observer is given by

$$
\begin{aligned}
\dot{\gamma}_{1} & =-k_{p} \gamma_{1}+\gamma_{2}+k_{p}\left(y-k_{p} y\right)+k_{i} y, \\
\gamma_{1}(0) & =\gamma_{1_{0}}, \\
\dot{\gamma}_{2} & =-k_{i} \gamma_{1}+k_{i}\left(y-k_{p} y\right), \\
\gamma_{2}(0) & =\gamma_{2_{0}} \\
\hat{\eta} & =\gamma_{1}+k_{p} y, \\
\hat{\eta}_{1} & =\gamma_{2}+k_{i} y .
\end{aligned}
$$

Now, we can use $\hat{\eta}$ to estimate the variables of interest $x_{1}$ and $x_{3}$ as follows. From expression (34) we can consider the following relationships:

$$
\begin{aligned}
& \hat{\eta}=\hat{x}_{1}+x_{3}, \\
& \dot{y}=\hat{\eta}-y .
\end{aligned}
$$

Deriving (40), we have

$$
\dot{\hat{\eta}}=\dot{\hat{x}}_{1}+\dot{x}_{3}=\dot{\hat{x}}_{1}-b y .
$$

On the other hand,

$$
\hat{\eta}=\gamma_{1}+k_{p} y
$$

such that

$$
\dot{\hat{\eta}}=\dot{\gamma}_{1}+k_{p} \dot{y} .
$$

From (42) and (44) we get

$$
\dot{\hat{x}}_{1}=\dot{\gamma}_{1}+k_{p} \dot{y}+b y \text {. }
$$

Substituting $\dot{\gamma}_{1}$ from (39) and (41) in (45), we have

$$
\begin{aligned}
\dot{\hat{x}}_{1}= & -k_{p} \gamma_{1}+\gamma_{2}+k_{p}\left(y-k_{p} y\right) \\
& +k_{i} y+k_{p}(\hat{\eta}-y)+b y .
\end{aligned}
$$

Then substitute (43) in (46) and simplify

$$
\begin{aligned}
\dot{\hat{x}}_{1} & =\gamma_{2}+k_{i} y+b y \\
& =\hat{\eta}_{1}+b y .
\end{aligned}
$$

Equation (47) can be solved with the Euler method,

$$
\begin{aligned}
\hat{x}_{1}(t+1) & =\hat{x}_{1}(t)+h\left[\hat{\eta}_{1}(t)+b y(t)\right] \\
\hat{x}_{1}(0) & =\hat{x}_{1_{0}}
\end{aligned}
$$


Finally, from (40) we can consider

$$
\hat{x}_{3}=\hat{\eta}-\hat{x}_{1} .
$$

Therefore, all variables of interest are estimated.

One can note that in this case synchronization and estimation occur simultaneously.

In what follows, consider a nonlinear system that describes the movement and the behaviour of the ship mass in a test of vertically landing over the surface of a planet with constant gravitational acceleration $g$ and no atmospheric resistance.

Example 5. Consider the system described by

$$
\begin{aligned}
\dot{x}_{1} & =x_{2}, \\
\dot{x}_{2} & =g-\frac{\sigma \alpha u}{x_{3}}, \\
\dot{x}_{3} & =-\sigma u-f, \\
y & =x_{1},
\end{aligned}
$$

where $x_{1}, x_{2}$ and $x_{3}$ are the vertical position, the descent velocity and mass of the ship, respectively. Here $u$ is a control signal, $\sigma$ is the relative velocity of ejection and $\alpha$ is a positive constant such that $\sigma \alpha$ is the maximal displacement of the motor breaking. Variable $f$ is an uncertainty that needs to be observed.

One can note that this system is nondifferentially flat due to

$$
\begin{aligned}
P\left(x_{1}\right) & =x_{1}-y=0, \\
P\left(x_{2}\right) & =x_{2}-\dot{y}=0, \\
P\left(x_{3}, \dot{x}_{3}\right) & =(\ddot{y}-g) x_{3}-\left(\dot{x}_{3}+f\right) \alpha=0 .
\end{aligned}
$$

System (50) can be written in the following form (immersion):

$$
\begin{aligned}
\dot{x}_{1} & =x_{2}, \\
\dot{x}_{2} & =g-\frac{\sigma \alpha u}{x_{3}}, \\
\dot{x}_{3} & =-\sigma u-f, \\
\dot{f} & =\Omega\left(x_{1}, x_{2}, x_{3}\right) . \\
y & =x_{1}
\end{aligned}
$$

Suppose that $\Omega$ is bounded, such that $\mathrm{H} 2$ is satisfied. The unknown variable $f$ satisfies $\mathrm{H} 1$ due to

$$
(f+\sigma u)(\ddot{y}-g)^{2}+\sigma \alpha u y^{(3)}=0
$$

In order to estimate $f$, consider the next PI observer

$$
\begin{aligned}
& \dot{\hat{f}}=k_{p f}(f-\hat{f})+\hat{f}_{1}, \\
& \dot{\hat{f}}_{1}=k_{i f}(f-\hat{f}) .
\end{aligned}
$$

Substituting (53), we have

$$
\begin{aligned}
& \dot{\hat{f}}=-k_{p f}\left(\sigma u+\frac{\sigma \alpha u y^{(3)}}{(\ddot{y}-g)^{2}}+\hat{f}\right)+\hat{f}_{1}, \\
& \dot{\hat{f}}_{1}=-k_{i f}\left(\sigma u+\frac{\sigma \alpha u y^{(3)}}{(\ddot{y}-g)^{2}}+\hat{f}\right) .
\end{aligned}
$$

Variables $y^{(3)}$ and $\ddot{y}$ are not available. Nevertheless, we can approximate them as $y^{(3)} \approx \ddot{\hat{x}}_{2}$ and $\ddot{y}=\dot{\hat{x}}_{2}$. Therefore, we have

$$
\begin{aligned}
& \dot{\hat{f}}=-k_{p f}\left(\sigma u+\frac{\sigma \alpha u \ddot{\hat{x}}_{2}}{\left(\dot{\hat{x}}_{2}-g\right)^{2}}+\hat{f}\right)+\hat{f}_{1}, \\
& \dot{\hat{f}}_{1}=-k_{i f}\left(\sigma u+\frac{\sigma \alpha u \ddot{\hat{x}}_{2}}{\left(\dot{\hat{x}}_{2}-g\right)^{2}}+\hat{f}\right),
\end{aligned}
$$

Let $\gamma_{1_{f}}$ and $\gamma_{2_{f}}$ be auxiliary variables satisfying $\mathrm{H} 3$,

$$
\begin{aligned}
\gamma_{1_{f}} & =\hat{f}-k_{p f} \frac{\sigma \alpha u}{\dot{\hat{x}}_{2}-g}, \\
\gamma_{2_{f}} & =\hat{f}_{1}-k_{i f} \frac{\sigma \alpha u}{\dot{\hat{x}}_{2}-g} .
\end{aligned}
$$

The corresponding derivatives are

$$
\begin{aligned}
& \dot{\gamma}_{1_{f}}=\dot{\hat{f}}+k_{p f} \ddot{\hat{x}}_{2} \frac{\sigma \alpha u}{\left(\dot{\hat{x}}_{2}-g\right)^{2}}, \\
& \dot{\gamma}_{2_{f}}=\dot{\hat{f}}_{1}+k_{i f} \ddot{\hat{x}}_{2} \frac{\sigma \alpha u}{\left(\dot{\hat{x}}_{2}-g\right)^{2}}
\end{aligned}
$$

Substituting (56) and (57) in 58), we have that the PI observer for $f$ is

$$
\begin{aligned}
\dot{\gamma}_{1_{f}}= & -k_{p f}\left(\sigma u+\gamma_{1_{f}}+k_{p f} \frac{\sigma \alpha u}{\dot{\hat{x}}_{2}-g}\right)+\gamma_{2_{f}} \\
& +k_{i f} \frac{\sigma \alpha u}{\dot{\hat{x}}_{2}-g} \\
\gamma_{1_{f}}(0)= & \gamma_{1_{f} 0}, \\
\dot{\gamma}_{2_{f}}= & -k_{i f}\left(\sigma u+\gamma_{1_{f}}+k_{p f} \frac{\sigma \alpha u}{\hat{\hat{x}}_{2}-g}\right) \\
\gamma_{2_{f}}(0)= & \gamma_{2_{f} 0}, \\
\hat{f}= & \gamma_{1_{f}}+k_{p f} \frac{\sigma \alpha u}{\dot{\hat{x}}_{2}-g}, \\
\hat{f}_{1}= & \gamma_{2_{f}}+k_{i f} \frac{\sigma \alpha u}{\dot{\hat{x}}_{2}-g} .
\end{aligned}
$$

As (59) involves the variable $\dot{\hat{x}}_{2}$, we have to to estimate it.

Consider the following PI observer for $x_{2}$ :

$$
\begin{aligned}
\dot{\hat{x}}_{2} & =k_{p x_{2}}\left(\dot{y}-\hat{x}_{2}\right)+\hat{x}_{2_{1}}, \\
\dot{\hat{x}}_{2_{1}} & =k_{i x_{2}}\left(\dot{y}-\hat{x}_{2}\right),
\end{aligned}
$$


where $x_{2}=\dot{y}$. Consider the following auxiliary variables

$$
\begin{aligned}
& \gamma_{1_{x_{2}}}=\hat{x}_{2}-k_{p x_{2}} y, \\
& \gamma_{2_{x 2}}=\hat{x}_{2_{1}}-k_{i x_{2}} y .
\end{aligned}
$$

The derivatives of the auxiliary variables are

$$
\begin{aligned}
& \dot{\gamma}_{1_{x 2}}=\dot{\hat{x}}_{2}-k_{p x_{2}} \dot{y} \\
& \dot{\gamma}_{2_{x 2}}=\dot{\hat{x}}_{2_{1}}-k_{i x_{2}} \dot{y} .
\end{aligned}
$$

Hence, substituting (60) and 610 in (62) we have

$$
\begin{aligned}
\dot{\gamma}_{1_{x 2}} & =-k_{p x_{2}}\left(\gamma_{1_{x 2}}+k_{p x_{2}} y\right)+\gamma_{2_{x 2}}+k_{i x_{2}} y \\
\gamma_{1_{x 2}}(0) & =\gamma_{1_{x 2_{0}}}, \\
\dot{\gamma}_{2_{x 2}} & =-k_{i x_{2}}\left(\gamma_{1_{x 2}}+k_{p x_{2}} y\right) \\
\gamma_{2_{x 2}}(0) & =\gamma_{2_{x 2_{0}}} \\
\hat{x}_{2} & =\gamma_{1_{x 2}}+k_{p x_{2}} y \\
\hat{x}_{2_{1}} & =\gamma_{2_{x 2}}+k_{i x_{2}} y .
\end{aligned}
$$

One can note that $\dot{y} \approx \hat{x}_{2}$. Therefore, from (62) we have

$$
\begin{aligned}
\dot{\hat{x}}_{2}= & -k_{p x_{2}}\left(\gamma_{1_{x 2}}+k_{p x_{2}} y\right) \\
& +\gamma_{2_{x 2}}+k_{i x_{2}} y+k_{p x_{2}} \hat{x}_{2} .
\end{aligned}
$$

Thus, $\dot{\hat{x}}_{2}$ can be estimated and therefore the variable of interest $f$ is obtained.

Remark 4. Expressions (59), 63) and (64) constitute a bank of observers for system (52).

\section{Numerical results}

In this section are presented numerical results of Examples 3-5.

For Example 3, we consider the initial conditions $\gamma_{1}(0)=0$ and $\gamma_{2}(0)=0$. The known variable is $u=$ $2 \sin (\pi t)$ and the unknown variable is $f=3 \sin (\pi t)$. The model-free-based PI observer gains were set to $k_{i}=15$ and $k_{p}=8$.

For Example 4, we consider the Chua system parameters $a=9.5, b=100 / 7, m_{0}=-8 / 7$ and $m_{1}=4 / 63$, which ensure a chaotic behavior, with initial conditions $x_{0}=(0.2,0.2,0.2)$. We consider $\gamma_{1}(0)=0$, $\gamma_{2}(0)=0$ and $\hat{x}_{1}(0)=0$. The model-free PI observer gains are set to $k_{i}=150$ and $k_{p}=25$.

Finally, for Example 5, we consider $g=1.63 \mathrm{~m} / \mathrm{s}^{2}$, $\sigma=50 \mathrm{~kg} / \mathrm{s}, \alpha=200 \mathrm{~m} / \mathrm{s}$ and the initial conditions $x_{0}=$ $(-700,0,1500)$. For the model-free PI observer consider $\gamma_{1_{x 2}}(0)=0, \gamma_{2_{x 2}}(0)=0, \gamma_{1_{f}}(0)$ and $\gamma_{2_{f}}(0)=0$, with $k_{p f}=49, k_{i f}=700, k_{p x_{2}}=6$ and $k_{i x_{2}}=25$. The control signal is $u=1$ and the uncertain variable is $f=$ $\exp (6 \sin (t))$
Numerical simulations show that the model-free-based PI observer is capable of performing a correct estimation of the unknown state variables for

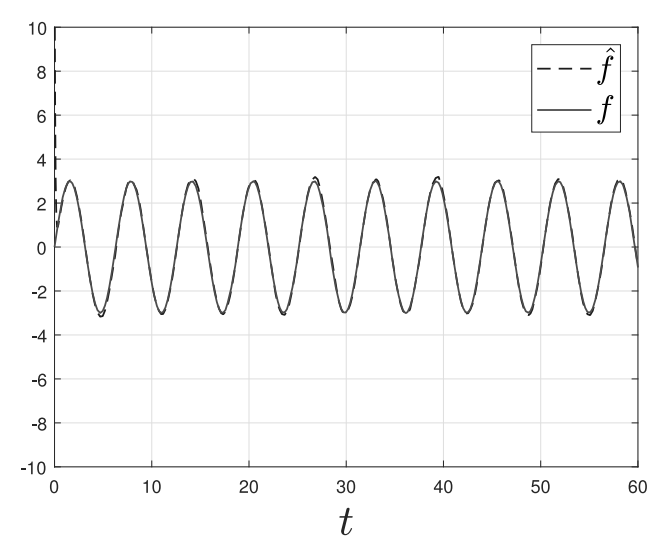

Fig. 1. Case 1: estimation of $f$ made by the PI observer.

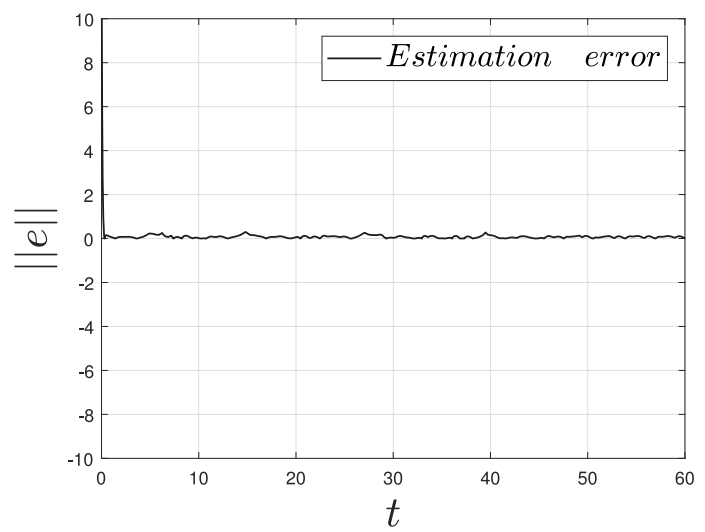

Fig. 2. Case 1: estimation error of the PI observer.

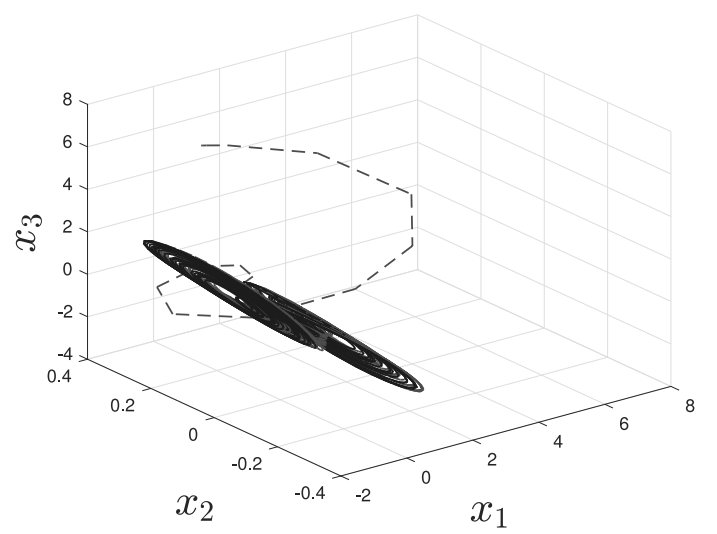

Fig. 3. Case 2: synchronization between the Chua system (solid line) and the PI observer (dotted line). 


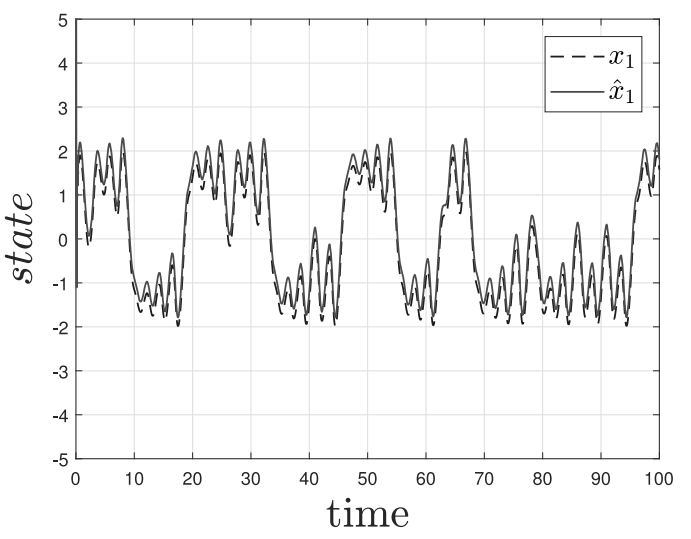

Fig. 4. Case 2: estimation of the state variable $x_{1}$ made by the PI observer.

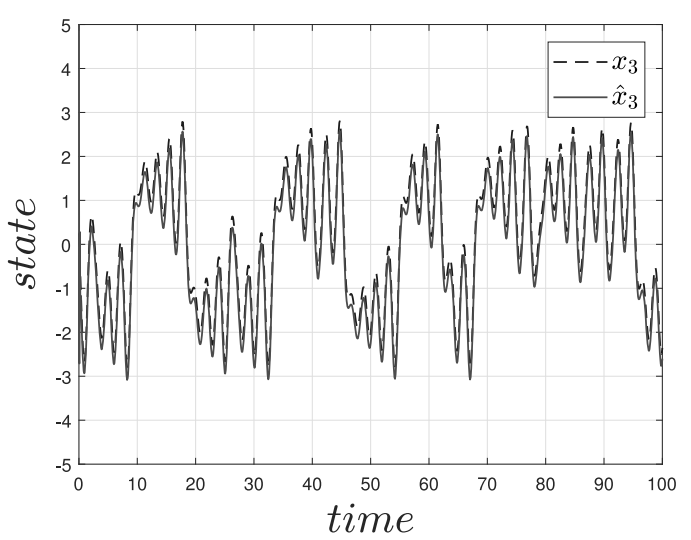

Fig. 5. Case 2: estimation of the state variable $x_{3}$ made by the PI observer.

all cases. In the case of the Chua system, it is possible to observe the synchronization phenomenon when the Chua state trajectory and the estimates of the PI observer along with the output, are plotted in the phase space of the system. Hence, it is proven that the estimation problem and the synchronization problem are solved simultaneously. On the other hand, Example 5 illustrates a situation where a bank of observers is obtained in order to estimate the variable of interest $f$.

\section{Conclusion}

It has been shown that the estimation problem for nondifferentially flat systems can be solved through differential polynomials of the output and with the right selection of auxiliary variables. As far as we know, the estimation problem for nondifferentially flat systems has not been solved in the literature yet. The observer proposed here is a free-model observer, which is a more natural way of proposing an observer since we usually

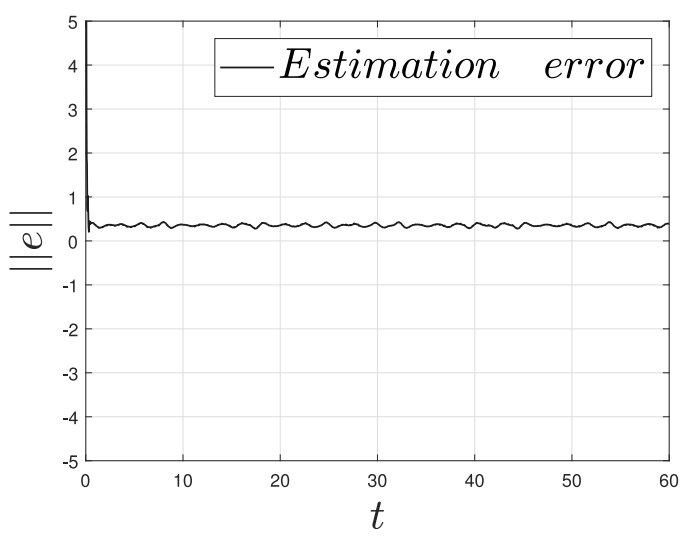

Fig. 6. Case 2: estimation error of the PI observer.

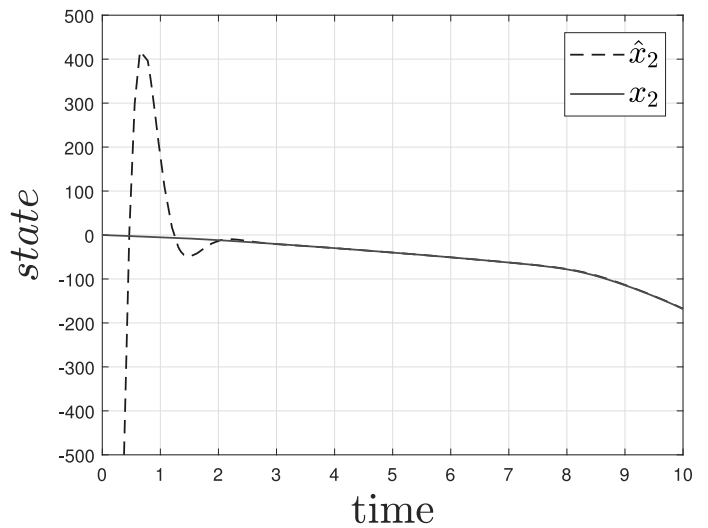

Fig. 7. Case 3: estimation of the state variable $x_{2}$ made by the PI observer.

do not have a copy of the system. Depending on the case, the variables of interest are estimated directly or indirectly from an auxiliary variable. This might lead to situations where the estimation problem requires an observer for each variable of interest (bank of observers). The type of convergence obtained is UUB (uniform ultimate boundedness). Lemma 1 shows the structure of the model-free-based PI observer for a relationship of the form (17). For a chaotic system, the estimation and the synchronization phenomenon between the system and the PI observer occur simultaneously. Finally, even this approach has been focused on nondifferential systems. It is worth to mentioning that the proposed methodology works for a larger class of systems.

\section{References}

Boutayeb, M., Darouach, M. and Rafaralahy, H. (2002). Generalized state-space observers for chaotic synchronization and secure communication, IEEE 
Transactions on Circuits and Systems I: Fundamental Theory and Applications 49(3): 345-349.

Claude, D., Fliess, M. and Isidori, A. (1983). Immersion directe et par bouclage d'un système non linéaire dans un linéaire, Comptes Rendus Des Seances De L'Academie Des Sciences 296(I): 237-240.

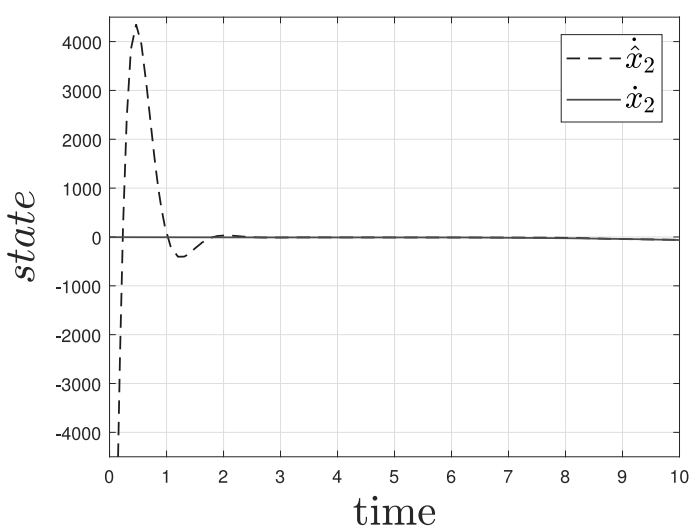

Fig. 8. Case 3: estimation of $\dot{x}_{2}$ made by the PI observer.

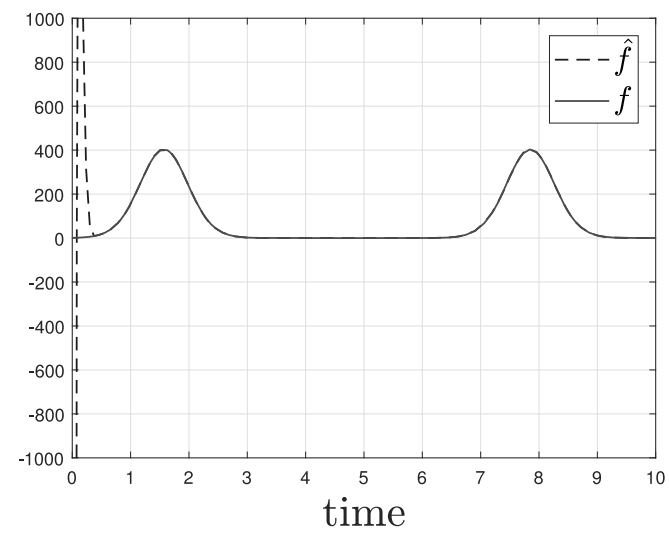

Fig. 9. Case 3: estimation of $f$ made by the PI observer.

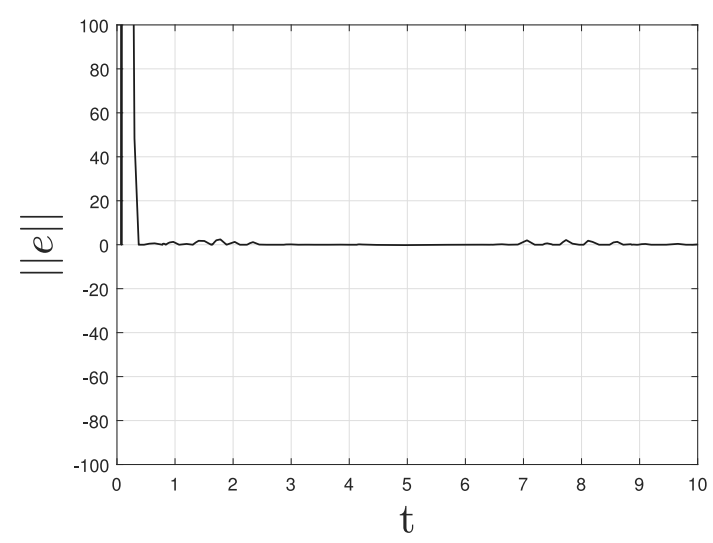

Fig. 10. Case 3: estimation error of the PI observer for $f$.
Corless, M. and Leitmann, G. (1981). Continuous state feedback guaranteeing uniform ultimate boundedness for uncertain dynamic systems, IEEE Transactions on Automatic Control 26(5): 1139-1144.

Gauthier, J.P., Hammouri, H. and Othman, S. (1992). A simple observer for nonlinear systems applications to bioreactors, IEEE Transactions on Automatic Control 37(6): 875-880.

Gensior, A., Woywode, O., Rudolph, J. and Guldner, H. (2006). On differential flatness, trajectory planning, observers, and stabilization for dc-dc converters, IEEE Transactions on Circuits and Systems I: Regular Papers 53(9): 2000-2010.

Karagiannis, D., Astolfi, A. and Ortega, R. (2005). Nonlinear stabilization via system immersion and manifold invariance: Survey and new results, Multiscale Modeling \& Simulation 3(4): 801-817.

Kolchin, E.R. (1973). Differential Algebra and Algebraic Groups, Academic Press, New York City, NY.

Layek, G. (2015). An Introduction to Dynamical Systems and Chaos, Springer, New York City, NY.

Martínez-Guerra, R. and Cruz-Ancona, C.D. (2017). Algorithms of Estimation for Nonlinear Systems, Springer, New York City, NY.

Martínez-Guerra, R., Cruz-Victoria, J., Gonzalez-Galan, R. and Aguilar-Lopez, R. (2006). A new reduced-order observer design for the synchronization of Lorenz systems, Chaos, Solitons \& Fractals 28(2): 511-517.

Martinez-Guerra, R. and Flores-Flores, J.P. (2018). Synchronization for a class of nondifferentially flat chaotic systems by means of a PI observer, 15th International Conference on Electrical Engineering, Computing Science and Automatic Control (CCE), Mexico City, Mexico, pp. 1-5, paper 25.

Martínez-Guerra, R., Gómez-Cortés, G. and Pérez-Pinacho, C. (2015). Synchronization of Integral and Fractional Order Chaotic Systems: A Differential Algebraic and Differential Geometric Approach with Selected Applications in RealTime, Springer, New York City, NY.

Martínez-Guerra, R., González-Galan, R., Luviano-Juárez, A. and Cruz-Victoria, J. (2007). Diagnosis for a class of non-differentially flat and Liouvillian systems, IMA Journal of Mathematical Control and Information 24(2): 177-195.

Martinez-Guerra, R. and Mendoza-Camargo, J. (2004). Observers for a class of Liouvillian and non-differentially flat systems, IMA Journal of Mathematical Control and Information 21(4): 493-509.

Martínez-Guerra, R. and Pérez-Pinacho, C.A. (2018). Advances in Synchronization of Coupled Fractional Order Systems: Fundamentals and Methods, Springer, New York City, NY.

Nijmeijer, H. and Mareels, I.M. (1997). An observer looks at synchronization, IEEE Transactions on Circuits and Systems I: Fundamental Theory and Applications 44(10): 882-890.

Pecora, L.M. and Carroll, T.L. (1990). Synchronization in chaotic systems, Physical Review Letters 64: 821-824. 
Pledgie, S.T., Hao, Y., Ferreira, A.M., Agrawal, S.K. and Murphey, R. (2002). Groups of unmanned vehicles: Differential flatness, trajectory planning, and control, IEEE International Conference on Robotics and Automation, ICRA'02, Washington, DC, USA, pp. 3461-3466.

Ritt, J.F. (1950). Differential Algebra, American Mathematical Society, New York City, NY.

Sira-Ramirez, H. (2002). A flatness based generalized PI control approach to liquid sloshing regulation in a moving container, American Control Conference, Anchorage, AK, USA, pp. 2909-2914.

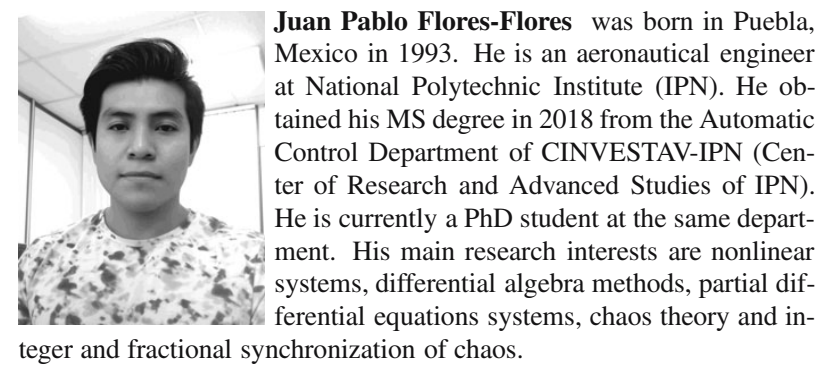

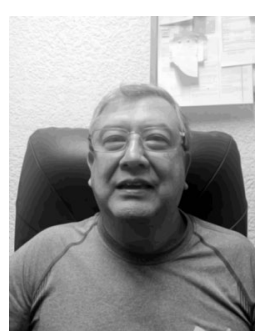

Rafael Martinez-Guerra was born in Mexico City in 1959. He obtained his $\mathrm{PhD}$ degree from Metropolitan Autonomous University in 1996. Currently, he is a researcher at the Automatic Control Department of CINVESTAV-IPN (Center of Research and Advanced Studies of IPN) and a member of the National System of Researchers (since 1992). He is the author and a co-author of more than 73 papers in international journals. He is also a co-author of 7 monographs. His main research interests are in the areas of nonlinear systems, differential geometric and differential algebraic methods, fractional nonlinear observers, fractional fault detection and diagnosis problem, secure communications, fractional synchronization and chaos.

Received: 30 November 2018

Revised: 16 April 2019

Accepted: 29 July 2019 